\title{
NANOSTRUCTURING AND HARDNESS INVESTIGATIONS OF THIN FILMS BY SCANNING FORCE MICROSCOPY
}

\author{
M. NoWICKI ${ }^{a}$, A. RICHTER ${ }^{b}$, R. RIES ${ }^{b}$ AND M. OsZWALDOW'SKI ${ }^{a}$ \\ ${ }^{a}$ Institute of Technical Physics, Poznan University of Technology \\ Piotrowo 3, 60-965 Poznań, Poland \\ ${ }^{b}$ Department of Physics and Technology, Technische Fachhochschule Wildau \\ Bahnhofstr. 1, 15745 Wildau, Germany
}

The scanning force microscope was used to scratch thin films and to write nanoscale pattern on surfaces as well as to perform nanoindentation for hardness measurements. Different thin film materials such as $\mathrm{C}_{60}$ films, diamond-like carbon, metals and semiconducting films have been investigated.

PACS numbers: $61.16 . \mathrm{Ch}, 68.35 .-\mathrm{p}$

\section{Introduction}

Manipulation of atoms or molecules with scanning probe microscopy and its application to the development of novel devices or novel memory systems have been studied extensively [1-4]. In particular, with a scanning tunneling microscope (STM) single atoms can be manipulated [2]. With scanning force microscopy (SFM), nanostructuring of Langmuir-Blodgett films as resist films have been examined [1] and nanofabrication of weak links in superconducting niobium films for SQUID devices has been verified [3]. Thus nanometer scale patterning was done successfully by scratching directly the sample surface with the tip. An advantage of the use of the SFM is its applicability even to non-conducting surfaces. Moreover it was demonstrated that nanomodification on surfaces can be obtained by an STM tip, which is externally laterally irradiated with short laser pulses under ambient conditions [4]. This technique is based on the local intensity enhancement of optical radiation close to a sharp tip.

Hardness investigations on ultrathin films of thickness in the range of 10 to $30 \mathrm{~nm}$ are of considerable interest with concern to protective coatings to increase for example the durability of magnetic disks [5]. The tip of the indentation body should indent only $10 \%$ of the film thickness to avoid the influence of the substrate. Forces generated with the tip on a cantilever within the SFM are favourable to 
measure the nanohardness of materials. Soft materials such as organic or molecular films and heterogeneous materials also profit from this method. Because of the small indented area a high lateral accuracy of the hardness in these materials is additionally possible.

It is the aim of this paper to report about our investigations of interaction between the SFM tip and various thin film surfaces with application to nanotechnology, that is to say nanostructuring and nanohardness.

\section{Experimental details}

$\mathrm{C}_{60}$ films are deposited by thermal evaporation in a vacuum chamber on different substrate materials with various thicknesses and a suitable substrate Ninperature of approximately $200^{\circ} \mathrm{C}$ [6]. Since surface roughness is an important factor in nanostructuring and nanohardness measurements the growth mode of the films was extensively studied. $\mathrm{C}_{60}$ films consist of molecules with a diameter of $1 \mathrm{~nm}$ and show a wide range of surface roughness $R_{\mathrm{a}}$ between molecular roughness of less than $R_{\mathrm{a}}=0.5 \mathrm{~nm}$ and $R_{\mathrm{a}}=8-10 \mathrm{~nm}$ depending on substrate materials and growth mode of the films. In this paper we only present results of smooth $\mathrm{C}_{60}$ films grown on mica.

Diamond-like carbon (DLC) films on silicon are deposited by laser ablation from a dense graphite target in a high vacuum chamber. These amorphous films do not contain hydrogen and have a considerable amount of $s p^{\dot{3}}$ bonding [7]. They are atomically flat with a surface roughness of less than $R_{\mathrm{a}}=0.05 \mathrm{~nm}$ and preferentially suited for multilayer X-ray mirrors with the demand of very sharp interfaces [8].

Metallic films on glass substrates are grown by thermal evaporation, whereas the semiconducting InSb films are deposited by flash evaporation [9]. Polycrystalline gold films could be obtained with a surface roughness of less than $0.8 \mathrm{~nm}$. The aluminium films produced in the same way are rougher with $R_{\mathrm{a}}=4.5 \mathrm{~nm}$ and have an additional thin oxide layer on top. The effect of the oxide layer can be observed with mutual changes of the morphology after scanning the tip over the surface several times. The polycrystalline InSb films consist of regular small crystals. Thus the surface roughness varies between $10 \mathrm{~nm}$ and $70 \mathrm{~nm}$. At low substrate temperatures flatter films can be obtained [9]. In this contribution only results of smooth films with approximately $10 \mathrm{~nm}$ surface roughness are presented.

Hardness indentation and nanostructuring are performed in a modified SFM Nanoscope III. The advantage of the SFM is based on the fact that the same tip on the cantilever is the device for mechanical surface modification in the contact operation mode and surface imaging after indentation or scratching. Thus, with high applied loading forces up to $1000 \mathrm{nN}$ nanoscale pattern are obtained in the film surface whereas the topography of the surface is obtained with the same tip at a loading force of 10-20 nN. This gives the possibility to measure the modified surface area directly. Different types of cantilevers and probe tips made from $\mathrm{Si}_{3} \mathrm{~N}_{4}$, $\mathrm{Si}$, and diamond single crystal pyramid have been tested. With the known spring constant of the cantilever and the knowledge of the force curve the applied load can be calculated. 


\section{Results}

In the macroscopic region hardness is determined by the applied normal load divided by either the curved surface area or the projected area of contact between the indenter and the material being tested under load. Hardness measurements on ultrathin films on a submicron scale using commercially available depth sensing instruments require a minimum thickness of $100 \mathrm{~nm}$. The hardness of a material is not only determined by the strength of the interatomic forces but also by the operative deformation mechanism. Therefore, the hardness value can vary with the microstructure and other structural constituents like voids, impurities, defects, textures etc.

Nanoindentation with a cantilever in a modified SFM is useful for testing the hardness of thin films. The key components for this technique are a special tip geometry and a tip hardness which is higher than the coatings to be investigated. Moreover, the tip and the cantilever should allow indentation as well as a proper imaging of the surface. Indentation cantilevers have a larger force constant, are also thicker, wider and longer than standard SFM cantilevers. The applied force is calculated from the force calibration curve, the projected indentation area is determined from the surface image. The hardness can then be computed as the quotient of indentation force and projected area. Other nanohardness techniques use a transducer to register the depth of the indenter with the loading force in dependence of the load [10]. In this case it is possible to distinguish also between elastic and plastic contributions.

First we used a conventional $\mathrm{Si}_{3} \mathrm{~N}_{4}$ tip with a force constant of only $0.38 \mathrm{~N} / \mathrm{m}$ to test a soft material. $\mathrm{C}_{60}$ molecules interact via van der Waals forces and form smooth fcc crystalline films on mica which are expected to have a very low hardness. Thus this novel carbon material was suitable for our investigations. For comparison, thick fullerite films of a few micrometers on mica and the bare mica substrate were analysed by standard microhardness measurements with applied forces of $1 \mathrm{mN}$ to $15 \mathrm{mN}$. Of course, with increasing load the hardness of the mica substrate is only measured. With small loading forces the microhardness of $\mathrm{C}_{60}$ films is $130 \mathrm{MPa}$. Now an epitaxially $100 \mathrm{~nm}$ thin $\mathrm{C}_{60}$ film was investigated by nanohardness measurements. With forces of $150-300 \mathrm{nN}$, indented areas between $900-2500 \mathrm{~nm}^{2}$ are obtained which results in nanohardness values of $167 \mathrm{MPa}$ to $120 \mathrm{MPa}$.

Hardness measurements of DLC films are especially interesting because of the expected high hardness and variety of hardness values in dependence on deposition techniques [11]. Additionally, the DLC films contain a high internal stress and can be grown only to a certain thickness up to $500 \mathrm{~nm}$ without additional stress reducing components. Their atomically flat surface is an ideal basis for nanomodification. We can report only about preliminary results since we could scratch the DLC film with a diamond tip, but because of the lack of the tapping mode in our device another tip has to be used for imaging. Very small indentation areas of 5-10 nm length were found with forces of about $1000 \mathrm{nN}$. Thus preliminary nanohardness values of maximum $40 \mathrm{GPa}$ are obtained which are less than the expected values estimated from $E$ modulus and density measurements [11]. 
Polycrystalline metallic films were examined with a silicon nitride tip and applied loading forces of more than $500 \mathrm{nN}$. The indents with these small forces are not very well visible and have dimensions not very much larger than the surface roughness. Nanohardness values for a $500 \mathrm{~nm}$ thick film of aluminium are about of $140 \mathrm{MPa}$ compared with microhardness values for aluminium of $120-450 \mathrm{MPa}$. Nanohardness values of a $700 \mathrm{~nm}$ thick gold film are of $138 \mathrm{MPa}$ compared to microhardness values of 100-200 MPa. Nanohardness investigations of InSb films are a problem because the indented area is not well visible in the rough surface and our applied loading forces are too small to obtain a larger indent. However relative to the metallic films the nanohardness is 2 to 3 times larger.
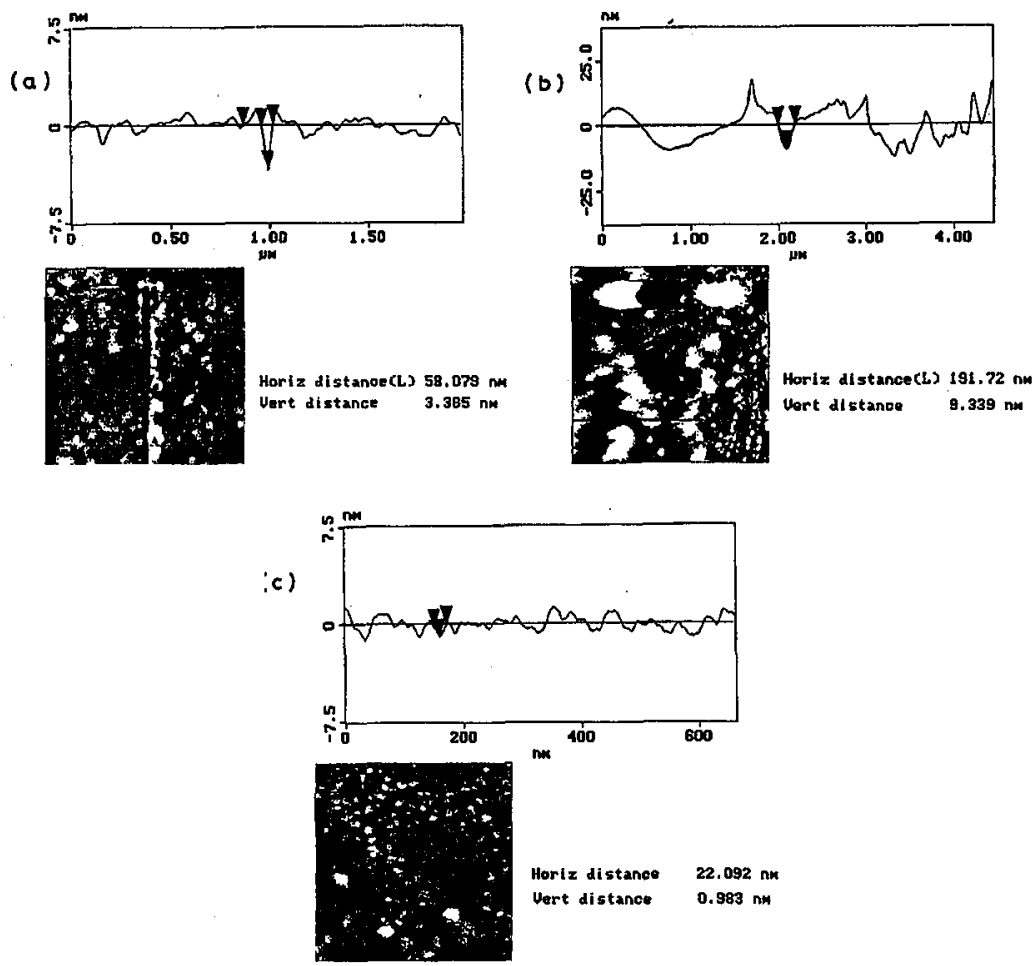

Fig. 1. SFM images with corresponding cross-section analysis of line scratches in $\mathrm{C}_{60}$ films (a), aluminium films (b) and gold films (c).

Nanostructuring of the above mentioned materials is also possible. Examples of scratched vertical lines are given in Fig. 1. The lines are better visible and can be found easier in the imaging process than the indents. Beside straight lines of different length and orientation to each other also other patterns can be written to fabricate miniaturized electronic structures as for example weak links. All lines were obtained with $\mathrm{Si}_{3} \mathrm{~N}_{4}$ pyramidal tips and a speed of $30 \mathrm{~nm} / \mathrm{s}$. Scratched ma- 
terial deposited along the nanostructures is visible especially on fullerite surfaces (Fig. 1a). The line width is dependent on the tip shape, but also on the material used. Lines of $20 \mathrm{~nm}$ to $200 \mathrm{~nm}$ could be generated (Fig. 1). The depth of the lines varies between 1 and $10 \mathrm{~nm}$ in the different materials. The relation between horizontal and vertical measures is about 20 and reflects roughly the geometry of the tip.

\section{Conclusion}

In conventional microhardness measurements an indentation of only $10 \%$ of the film thickness should be used to avoid the influence of the underlaying substrate. Therefore, new measurement techniques are required to characterize ultrathin and hard films correctly. It was possible to determine nanohardness values ranging from a soft material such as fullerite films with $0.12 \mathrm{GPa}$ up to hard diamond-like carbon films with $40 \mathrm{GPa}$. A correlation of hardness values in the nanometer regime and the micrometer regime is basically possible and reveals the same trend. The method can be also extended to the investigation of the local hardness of inhomogeneous multiphase structures. However, the method should be improved to higher accuracy in determination and measuring the load of the indentation force.

It could be shown that nanostructuring with different line width and depth is possible in a variety of materials. To improve the present nanolithography process, further studies of the force required to remove the film, i.e. the wear process will be necessary. Also the mechanism for the deposition of the scratched material on the tip should be investigated in more detail. Influence of scratch speed and frequency as well as repeated writing are important to generate nanopattern.

\section{References}

[1] H. Takano, M. Fujihira, Thin Solid Films 273, 312 (1996).

[2] R. Czajka, A. Kasuya, A. Wawro, N. Horiguchi, Y. Nishina, Surf. Rev. Lett. 3, 961 (1996).

[3] C. Hahn, T. Matsuyama, U. Merkt, R. Wiesendanger, Appl. Phys. A 62, 289 (1996).

[4] J. Jergsch, F. Demming, K. Dieckmann, Appl. Phys. A 64, 29 (1997).

[5] G. Persch, Ch. Born, B. Utesch, Appl. Phys. A 59, 29 (1994).

[6] A. Richter, R. Ries, K. Szulszewsky, B. Pietzak, R. Smith, Surf. Sci.1997, accepted for publication.

[7] A. Richter, M. Klose, Opt. Laser Technol. 24, 215 (1992).

[8] H. Mai, W. Pompe, Appl. Surf. Sci. 54, 215 (1992).

[9] M. Oszwałdowski, T. Berus, Phys. Status Solidi A 161, 143 (1997).

[10] B. Richarz, T. Fries, Mess- und Prüftechnik 50, 726 (1996).

[11] D. Schneider, T. Schwarz, H.-J. Scheibe, M. Panzner, Thin Solid Films 295, 107 (1997). 